\title{
Hot electron plasmas trapped in helical magnetic surfaces
}

\author{
H. Himura • H. Wakabayashi • Y. Yamamoto • \\ A. Sanpei $\cdot$ S. Masamune $\cdot$ M. Isobe . \\ S. Okamura $\cdot$ K. Matsuoka
}

Published online: 3 July 2007

(C) Springer Science + Business Media B.V. 2007

\begin{abstract}
Experimental studies on nonneutral (pure electron) plasmas of finite temperature, trapped in helical closed magnetic surfaces have been conducted. The helical electron plasmas are produced with thermal electrons launched from the outside of the last closed flux surface (LCFS). About $150 \mu \mathrm{s}$ after the electron injection, the plasmas reach equilibrium state. Around the LCFS, a steep gradient of plasma space potential $\phi_{S}$ is formed. The corresponding radial electric field is about $2.5 \mathrm{kV} / \mathrm{m}$. On the other hand, around the magnetic axis of helical magnetic surfaces, $\phi_{s}$ is almost constant, indicating that there are little electrons there. The volumeaveraged electron density is on the order of $10^{13} \mathrm{~m}^{-3}$, smaller than the Brillouin density limit. The confinement time seems to be limited by a disruptive instability, and is so far about $1.5 \mathrm{~ms}$.
\end{abstract}

Keywords Toroidal nonneutral plasmas $\cdot$ Helical magnetic surfaces

PACS 52.27.Jt • 52.27.Aj $\cdot 52.55 . \mathrm{Hc} \cdot 52.70 . \mathrm{Ds}$

\section{Introduction}

While nonneutral plasmas trapped in purely toroidal magnetic fields have been studied intensively for five decades [1], nonneutral plasmas confined in toroidal magnetic surfaces without any electric fields have just been investigated [2, 3]. The method of confining electrically nonneutral plasmas in closed magnetic surfaces

H. Himura ( $₫)$ Y Y. Yamamoto · A. Sanpei · S. Masamune Department of Electronics, Kyoto Institute of Technology, Kyoto 606-8585, Japan e-mail: himura@kit.ac.jp

H. Wakabayashi · M. Isobe · S. Okamura · K. Matsuoka National Institute for Fusion Science, Gifu 509-5292, Japan e-mail: isobe@nifs.ac.jp 
Fig. 1 A picture of the $\mathrm{LaB}_{6}$ emitter

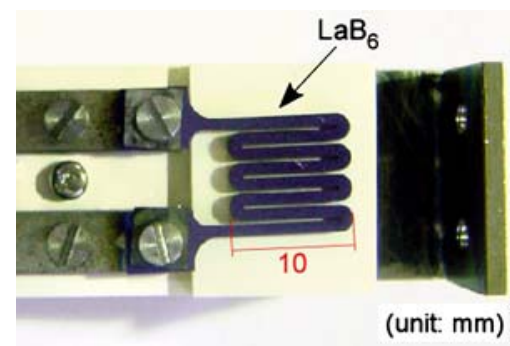

offers a possibility of reproportioning electrons and ions inside magnetic surfaces because the field is completely closed. This property may be applied to produce various fascinating plasmas such as two-fluid plasmas or electron-positron plasmas which have been studied mainly theoretically. The Compact Helical System (CHS) machine [4] is one of middle-sized stellarator devices for fusion plasmas, which can thus precisely form helical magnetic surfaces. A schematic drawing of CHS can be found in $[4,5]$. Using the machine, we have studied helical nonneutral plasmas having hot electron temperature $T_{e}$ up to $\sim 250 \mathrm{eV}$. In order to produce the plasma on CHS, thermal electrons have been injected via stochastic magnetic layer [5, 6]. Large space potential of the produced helical nonneutral plasma has been measured with a high-impedance emissive probe [7]. At the first series of experiments, we have successfully produced hot helical nonneutral plasmas on CHS. In this contributed paper, we present experimental results on basic properties of CHS helical nonneutral (electron) plasmas.

\section{Properties of helical electron plasmas on CHS}

Experiments have been conducted on CHS whose major $R$ and average minor radii $\bar{r}$ are 1.0 and $0.2 \mathrm{~m}$, respectively. The key parameter which identifies the configuration is the magnetic axis $R_{a x}$ that is usually fixed at $R=101.6 \mathrm{~cm}$. For this case, the stochastic magnetic region (SMR) $[5,6]$ is present where the LCFS is completely detached from the vacuum chamber. Thus, helical magnetic surfaces are electrically isolated. The typical magnetic field strength $B$ is $0.9 \mathrm{kG}$ at $R_{a x}$, which yields $\rho_{e} \sim$ $1.3 \mathrm{~mm}$ when the maximum speed of electrons is $v_{e} \sim 2 \times 10^{7} \mathrm{~m} / \mathrm{s}$ for $e V_{a c c} \sim 1.2 \mathrm{keV}$, where $\rho_{e}, v_{e}$, and $e V_{a c c}$ are the Larmor radius, the electron velocity, and the beam energy, respectively.

Electrons are injected from a diode-type electron gun (henceforth called e-gun) which uses a $\mathrm{LaB}_{6}$ emitter as the cathode. Figure 1 shows a picture of the $\mathrm{LaB}_{6}$ emitter. Values of beam current $\left(I_{b}\right)$ and $e V_{a c c}$ of the injected electrons can be varied. The e-gun is installed in the equatorial plane at $z=0$ and the cathode is placed in the SMR, usually at $r \sim 117.5 \mathrm{~cm}$ which is about $2 \mathrm{~cm}$ outside the LCFS. Nevertheless, substantial penetration of the injected electrons is observed in $\sim 100 \mu \mathrm{s}[5,6]$ and helical electron plasmas are produced inside magnetic surfaces.

\subsection{Plasma space potential $\phi_{S}$}

Plasma space potential $\phi_{s}$ are measured with the high-impedance emissive probe, and the data can be obtained at two different cross sections along the $r$ and $z$ axes on 
Fig. 2 A typical data of $\phi_{s}(r)$ and $\phi_{s}(z)$ for the case of $V_{\text {acc }}=1.2 \mathrm{kV}$. The solid and dashed curves correspond to $E_{z}$ and $E_{r}$ calculated from $E_{\alpha}=-\nabla_{\alpha} \phi_{s}(\alpha)$, respectively
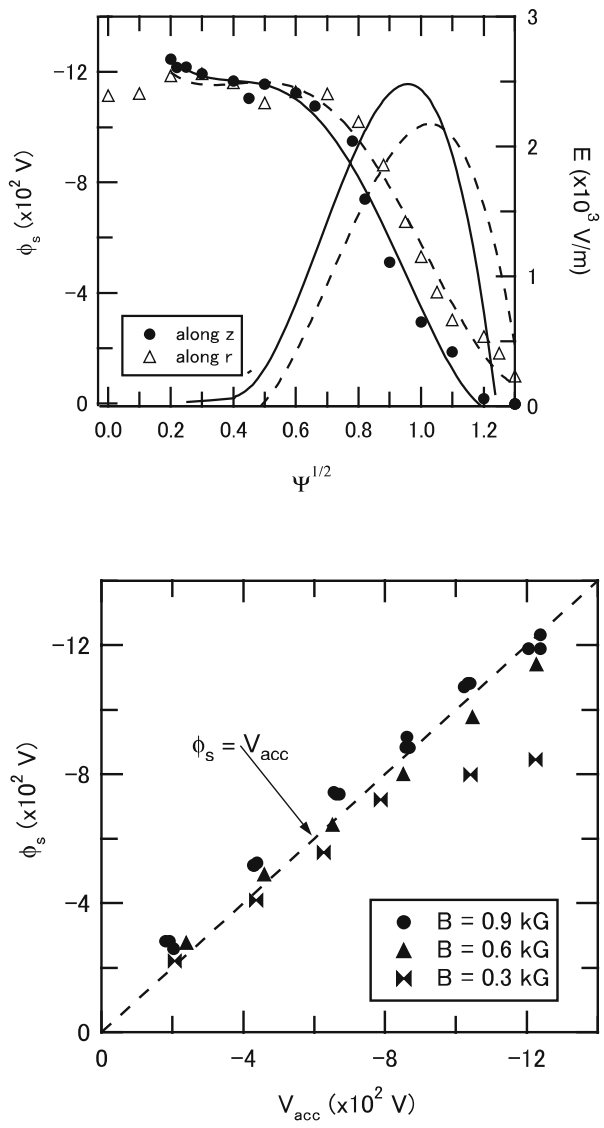

Fig. 3 The maximum $\phi_{s}$ at $R_{a x}$ plotted against $e V_{a c c}$

CHS. Here, we use a cylindrical coordinate measured from the center of the torus. Figure 2 shows a typical data of $\phi_{s}(r)$ and $\phi_{s}(z)$ for $V_{a c c}=1.2 \mathrm{kV}$. The horizontal axis is shown in $\Psi^{1 / 2}$ where $\Psi^{1 / 2}=0$ and 1 correspond to the $R_{a x}$ and LCFS, respectively. As recognized, large negative potential of $\phi_{s}$ is formed in magnetic surfaces. The corresponding electric field $E$ is also depicted in Fig. 2, which indicates that the maximum value of $E$ is about $3 \mathrm{kV} / \mathrm{m}$ at LCFS.

The value of $\phi_{s}$ achieves its maximum in the neighbourhood of $R_{a x}$. Figure 3 shows $\phi_{s}$ at $R_{a x}$ measured against $e V_{a c c}$ for cases of $B=0.9,0.6$, and $0.3 \mathrm{kG}$. As seen from the plotted data in Fig. 3, $\phi_{s}$ increases significantly with $V_{a c c}$. In addition, stronger $B$ results in the larger $\phi_{s}$. These results indicates that the maximum $\phi_{s}$ is limited by both $e V_{a c c}$ and the strength of $B$. The dependence on $e V_{a c c}$ can roughly be understood by invoking energy conservation for the injected electron. Assuming that the electron drift (rotation) speed is $\left(-\nabla \phi_{s}\right) / B$, it is calculated to be $\sim 10^{5} \mathrm{~m} / \mathrm{s}$. As will be shown, this value is much smaller than the electron thermal speed $v_{t h}\left(>10^{6} \mathrm{~m} / \mathrm{s}\right)$. Then, $e \phi_{s}=e V_{a c c}-\left(3 \kappa T_{e} / 2\right)-\omega P \simeq e V_{a c c}-\left(3 \kappa T_{e} / 2\right) \leq e V_{a c c}$ [8], where $3 \kappa T_{e} / 2$ and $\omega P$ are thermal and drift (rotation) energies, respectively. On the other hand, the dependence on $B$ is probably due to better confinement of electrons in the stronger $B$. 
Fig. 4 Typical data of $n_{e}(z)$ for $B=0.9 \mathrm{kG}$ and $V_{a c c}=$ $600 \mathrm{~V}$

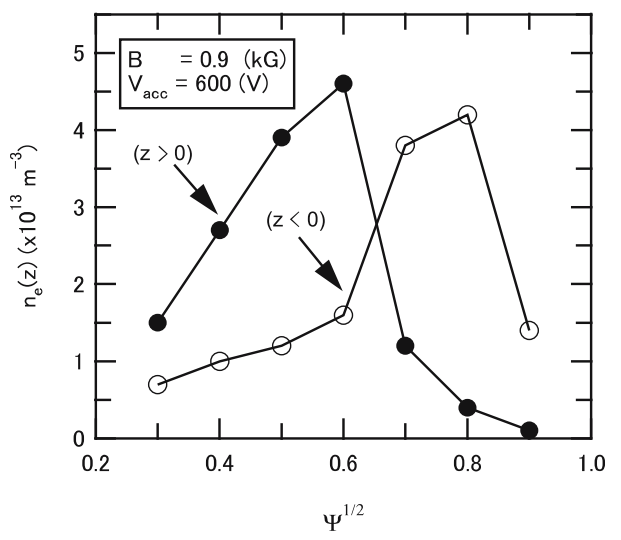

\subsection{Electron density $n_{e}$ and temperature $T_{e}$}

The current-voltage $\left(I_{e}-V_{p}\right)$ characteristics are also measured at each magnetic surface using the same emissive probe. Regarding with $n_{e}$, it is obtained from $I_{e}\left(\sim e n_{e} v_{t h} S\right)$ at $V_{p}=\phi_{s}$, where $\phi_{s}$ has been pre-measured just before the $I_{e}$ measurement, where $S$ is the probe area. All other contributions to $I_{e}$ except $v_{t h}$ are ignored, because $v_{t h}$ is much faster for the presented hot electron plasmas, as mentioned. Figure 4 shows $n_{e}(z)$ for $B=0.9 \mathrm{kG}$ and $V_{a c c}=600 \mathrm{~V}$. As seen from the plotted data, values of $n_{e}$ are on the order of $10^{13} \mathrm{~m}^{-3}$ in magnetic surfaces. Meanwhile, as explained in Fig. 2, the value of $\phi_{s}$ has decreased monotonically at $\Psi^{1 / 2} \geq 0.7$, that is, near the plasma boundary. Thus, if we apply $\nabla^{2} \phi_{s}=e n_{e} / \epsilon_{0}$ to the region, the experimental value of $n_{e}$ is inferred to be $10^{11-13} \mathrm{~m}^{-3}$ for $\phi_{s} \sim 1 \mathrm{kV}$, which is consistent with the measured $n_{e}$ there.

Here, one notes that unlike for fusion plasmas, $n_{e}$ is non-constant on each magnetic surface. In fact, such a non-uniformity can be recognized also in $\phi_{s}$. Although the detail is now under reviewed [9], these results confirm a fairly recent theoretical prediction [10] on equilibria of toroidal nonneutral plasmas confined in magnetic surfaces. In fact, by a dimensional analysis of the theory, the variation of $\phi_{s}$ on magnetic surfaces could be on the same order of $T_{e} / e$. Figure 5 shows a preliminary result of $T_{e}(z)$ for $V_{a c c}=600 \mathrm{~V}$ and $B=0.9 \mathrm{kG}$. As seen from the data, $T_{e}$ is in the range between 40 and $250 \mathrm{eV}$. The value of $T_{e}$ approximately corresponds to the observed variations of $\phi_{s}$ which are $\leq 150 \mathrm{~V}$. Similar consideration hold also for the $V_{a c c}=1 \mathrm{kV}$ case in which $T_{e} \leq 250 \mathrm{eV}$ and the difference in $\phi_{s} \leq 350 \mathrm{~V}$.

\subsection{Confinement time $\tau_{N}$}

An estimate of the confinement time $\tau_{N}$ of helical electron plasmas can be obtained from the signal of $I_{p}$ placed at the LCFS. Typical data are shown in Fig. 6, which indicates that $I_{p}$ persists for $\sim 1.5 \mathrm{~ms}$ after the electron injection is turned off at $t \sim 1.4 \mathrm{~ms}$. Thus, this result infers that $\tau_{N}$ is also about $1.5 \mathrm{~ms}$ for this shot. In fact, in most shots, $\tau_{N}$ is limited by a disruptive instability whose spike appears at the end of the duration of $I_{p}$, as seen at $t \sim 3 \mathrm{~ms}$ in Fig. 6. Although the details are still 
Fig. 5 A preliminary result of $T_{e}(z)$ for $V_{a c c}=600 \mathrm{~V}$ and $B=0.9 \mathrm{kG}$

Fig. 6 Typical signals of $I_{p}$ measured at the LCFS, with the electron injection current from the e-gun
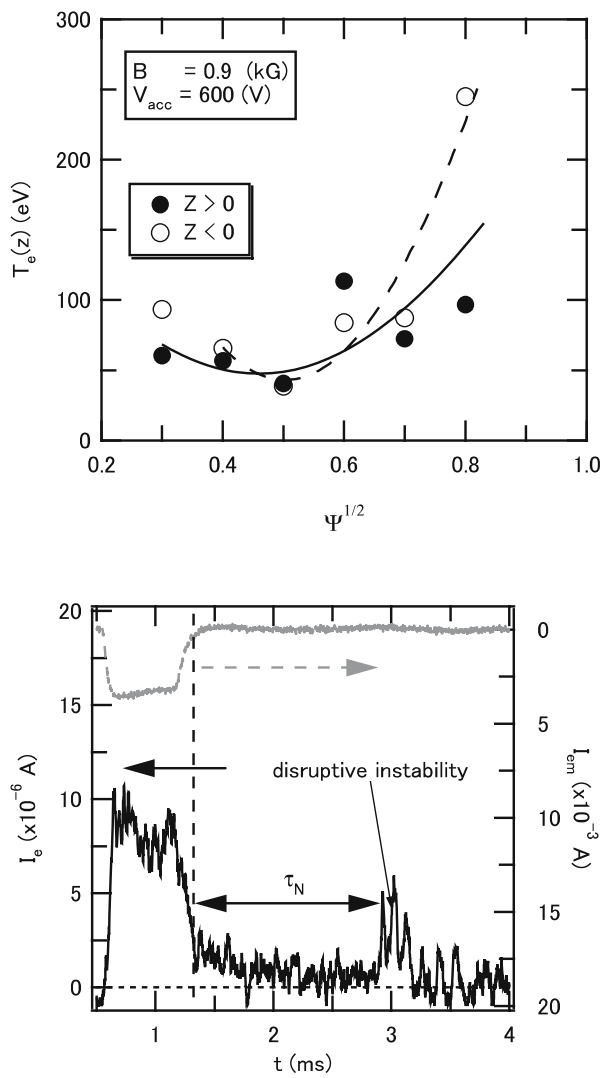

unknown, recent experiments have accumulated many data on the observed spike signals to address the instability of helical nonneutral plasmas.

Finally, since $\tau_{N}$ is approx. $1 \mathrm{~ms}$, the transport coefficient in experiments $D_{\text {exp }}$ is calculated to be $\sim \bar{r} / 2 \tau_{N} \sim 10 \mathrm{~m}^{2} / \mathrm{s}$. In most cases of helical neutral plasmas, the dominant transport mechanism is helically trapped particle (HTP) loss [11]. However, this does not account for helical electron plasmas. This is because the speed of HTP can be estimated as $m_{e} v_{e \perp}^{2} /\left(e B R_{h}\right) \sim 3 \times 10^{4} \mathrm{~m} / \mathrm{s}$, where $R_{h}$ is the radius of curvature for $\mathbf{B}$. Thus, the coefficient $D_{h}$ due to the HTP loss is approximately $\sim 10^{6} \mathrm{~m}^{2} / \mathrm{s}$, which is too large to explain $D_{\text {exp }}$. On the other hand, the coefficient due to radial electric field $D_{E}[11]$ is about $10 \mathrm{~m}^{2} / \mathrm{s}$, which seems to be same order as $D_{\text {exp }}$.

Acknowledgements The authors are grateful to the CHS group for performing this research on CHS. This work is performed under auspices of the NIFS CHS Research Collaboration.

\section{References}

1. Daugherty, J.D., Eninger, J.E., Janes, G.S.: Experiments on the injection and containment of electron clouds in a toroidal apparatus. Phys. Fluids 12, 2677 (1969)

2. Nakashima, C., Yoshida, Z., Himura, H., et al.: Injection of electron beam into a toroidal trap using chaotic orbits near magnetic null. Phys. Rev. E 65, 036409 (2002) 
3. Pedersen, T.S.: First non-neutral plasmas in the Columbia non-neutral torus. In: Stellarator news, vol. 99, p. 3 (2005)

4. Nishimura, K., Matsuoka, K., Fujiwara, M., et al.: Compact helical system physics and engineering design. Fus. Technol. 17, 86 (1990)

5. Himura, H., Wakabayashi, H., Fukao, M., et al.: Observation of collisionless inward propagation of electrons into helical vacuum magnetic surfaces via stochastic magnetic fields. Phys. Plasmas 11, 492 (2004)

6. Himura, H., Wakabayashi, H., Fukao, M., et al.: Experiments on injecting electrons into helical magnetic field configuration. IEEE Trans. Plasma Sci. 32, 510 (2004)

7. Himura, H., Fukao, M., Wakabayashi, H., Yoshida, Z.: Filament size of floating-emissive probe for low density plasmas with large space potential. 74, 4658 (2003)

8. Himura, H., Nakashima, C., Saito, H., Yoshida, Z.: Probing of flowing electron plasmas. Phys. Plasmas 8, 4651 (2001)

9. Himura, H., Wakabayashi, H., Yamamoto, Y., et al.: Experimental verification of non-contant potential and density on magnetic surfaces of helical nonneutral plasmas. Phys. Plasmas 14, 022507 (2007)

10. Pedersen, T.S., Boozer, A.H.: Confinement of nonneutral plasmas on magnetics surfaces. Phys. Rev. Lett. 88, 205002 (2002)

11. Wakatani, M.: Stellarator and heliotron devices, p. 271. Oxford University Press, New York (1998) 